\title{
Venous Thromboembolic Disease after Lung Transplantation: Special Focus on Sirolimus
}

\author{
Rajiv Lingaraju ${ }^{1}$, Nancy P Blumenthal ${ }^{1}$, Jim Mendez ${ }^{1}$, Olympia Hadjiliadis ${ }^{2}$, James C. Lee ${ }^{1}$, \\ Vivek N. Ahya ${ }^{1}$, Jason D. Christie ${ }^{1}$, Robert M. Kotloff ${ }^{1}$, Alberto Pochettino ${ }^{1}$ and Denis Hadjiliadis ${ }^{*}, 1$ \\ ${ }^{1}$ Lung Transplant Program, University of Pennsylvania, Philadelphia, PA, 19104, USA \\ ${ }^{2}$ Department of Mathematics, City University of New York, Brooklyn College, Brooklyn, NY, USA
}

\begin{abstract}
Background: Venous thromboembolism (VTE) is common after lung transplantation. We aimed to review our experience with VTE in lung transplant recipients, while paying attention to the use of sirolimus, a novel immunosuppressive agent that may have prothrombotic effects. Methods: A retrospective review of lung transplant recipients at the University of Pennsylvania from 2000 to 2006 was performed. Demographic data, acute and chronic rejection and survival were included. Clinically apparent VTE episodes and sirolimus utilization were noted. Results: $59 / 278(21.2 \%)$ of patients developed VTE. There were no baseline differences in patients with and without VTE. Patients more than three months post-transplant had a higher rate of VTE on sirolimus vs. off $(1.98 \% v s .0 .32 \%$ per month on vs. off sirolimus; $\mathrm{p}<0.001)$. Patients with VTE after lung transplantation had worse survival $(\mathrm{p}=0.001)$. Conclusions: VTE is a common complication after lung transplantation and patients with VTE have worse post-transplant survival. Sirolimus is associated with increased rate of VTE.
\end{abstract}

Keywords: Lung transplantation, venous thromboembolism, sirolimus.

\section{INTRODUCTION}

Lung transplantation remains an accepted therapy for patients with end-stage respiratory disease [1]. Unfortunately, post-transplant complications, particularly acute rejection and bronchiolitis obliterans syndrome, remain frequent, and median survival is approximately five years [2]. The risks of acute and chronic rejection necessitate immunosuppressive therapy, typically consisting of a calcineurin inhibitor (cyclosporine or tacrolimus), a purine synthesis inhibitor (azathioprine or mycophenolate mofetil), and a corticosteroid (prednisone) [2]. A well-documented and undesired side effect of the calcineurin inhibitors is nephrotoxicity, which can be severe and debilitating in transplant recipients [3].

Sirolimus is a macrolide approved for immunosuppression in solid-organ transplant recipients [4]. It is structurally similar to tacrolimus, but functions by inhibiting the mammalian target of rapamycin (mTOR) rather than calcineurin [4]. Sirolimus is relatively non-nephrotoxic, a major advantage over the calcineurin inhibitors [4]. Uniquely, however, sirolimus potentiates nephrotoxicity when used in conjunction with calcineurin inhibitors [4]. However, it was shown that sirolimus is not devoid of undesired side effects. Experience with sirolimus in lung transplantation has been associated with airway anastomotic dehiscence, and sirolimus use in liver transplantation has led to reports of delayed wound healing and hepatic artery thromboses [5-9]. Patients

*Address correspondence to this author at the Pulmonary and Critical Care, University of Pennsylvania, Lung Transplantation Program, Adult Cystic Fibrosis Program, 835W Gates Building, Hospital of the University of Pennsylvania, 3600, Spruce Street, Philadelphia, PA, 19104, USA; Tel: 215615-3871; Fax: 215-662-3226; E-mails: Denis.Hadjiliadis@uphs.upenn.edu, Hadjiliadisdenis@hotmail.com with coronary artery disease who were treated with sirolimus-eluting stents also showed a modest increase in thrombosis [10]. Meanwhile, cardiac transplant recipients treated with everolimus, an analog of sirolimus, showed low rates of vasculopathies, and sirolimus has not been associated with thromboembolic events in renal transplant recipients $[11,12]$.

Deep venous thrombosis and pulmonary embolism are common conditions after lung transplantation and can contribute to reduced post-transplant survival [13-15]. The effect of sirolimus on thrombosis in lung transplant recipients has not been addressed. This paper focuses on the effects of sirolimus on venous thromboembolic disease after lung transplantation at a single center. Some aspects of this paper have been presented as an abstract at the American Thoracic Society International Conference in May 2008 [16].

\section{MATERIALS AND METHODOLOGY}

A retrospective cohort study of lung transplant recipients from January 2000 through December 2006 at the University of Pennsylvania was performed. There were 268 first lung transplant recipients, 4 re-transplant lung recipients, 5 first heart-lung transplant recipients and 1 liver-lung transplant recipient. All patients were included in the primary analysis for VTE, re-transplants counted as separate events. In a second analysis, re-transplants and multiple organ transplants were excluded and the follow-up time was censored at the time of re-transplant for patients that received one. In that analysis, patients already on treatment for VTE were excluded and only patients surviving more than three months were included (since this is the population that was "at risk" of receiving sirolimus and VTE rates decrease significantly after the first three months post-transplant). 
Age, gender, type of transplant and pre-transplant diagnosis were collected for all patients and utilized for multivariable survival analysis. In addition, information on use of coumadin or low molecular weight heparin in treatment doses was recorded as well as the reason for their use. Episodes of deep venous thrombosis (DVT) and pulmonary embolism (PE) were noted after reviewing charts, medications and radiology records. No screening for VTE episodes was performed. All episodes were clinically apparent. Utilization, timing and length of sirolimus were recorded. Finally, presence of acute and chronic rejection and survival were obtained. The acute rejection score (ARS) was utilized as a composite measure to quantify the acute rejection episodes in the first six months after transplant and it is calculated by adding the A score of each rejection episode. This score has been utilized in the past to quantify acute rejection after lung transplantation [17].

Immunosuppression included tacrolimus, prednisone and azathioprine or mycophenolate mofetil (between 2002 and 2003). Some patients received sirolimus as part of a clinical trial (they were at least three months post-transplant) [18]. Acute rejection episodes were treated with pulse intravenous corticosteroids for three days (500-1000mg of methylprednisolone per day) or with oral prednisone taper, depending on the severity of acute rejection. All patients received Pneumocystis jiroveci prophylaxis with sulfamethoxazole/ trimethoprim or other medications. Antifungal and antiviral prophylaxis changed through the years as new medications became available. Current regimens include ganciclovir or valganciclovir for cytomegalovirus and voriconazole for high-risk patients for antifungal prophylaxis. Surveillance bronchoscopies with biopsies happen at 6 weeks, 3 months, 6 months and 12 months post-transplant, beginning in 1998. Clinically indicated bronchoscopy is also performed when patients have unexplained changes in their pulmonary status (clinical, radiographic and or spirometric). More detailed information on immunosuppressive and antibiotic prophylaxis protocols at our center have been published previously [19]. Patients received subcutaneous heparin for DVT prophylaxis after their transplant operation if there was no life-threatening bleeding, until discharge and on all subsequent admissions; this was modified accordingly in cases of allergy to heparin. In addition, aspirin $325 \mathrm{mg}$ daily was given during the transplant admission. DVT and PE were treated with intravenous heparin followed by coumadin or low molecular weight heparin for 3 to 6 months depending on the timing of the VTE episode.

Statistical analysis comparing the pre-transplant diagnoses of lung transplant recipients with and without VTE was performed with the Fisher's exact test. Survival estimates were calculated using the Kaplan-Meier method and groups were compared using the log-rank test. VTE-free time was similarly analyzed. Multivariable survival analysis was performed by utilizing the Cox proportional hazards method. Continuous variables were compared using the Student t-test or its non-parametric equivalent. Multivariable analysis was performed using logistic regression for patients developing VTE after the first 3 months post-transplant and included demographic variables as well as the acute rejection score. The SAS 9.1.3 (Cary, NC) statistical software was utilized for all analyses [20].

\section{RESULTS}

There were 59 patients with episodes of VTE in 278 transplant recipients during the study period (21.2\%). 20/59 (33.9\%) patients had a diagnosis of PE, while the rest only had only a diagnosis of DVT. Baseline characteristics of patients with and without VTE were not different and can be seen in Table 1. 29/59 (49.2\%) episodes of VTE occurred in the first three months post-transplant, for a rate of 29/278 $(10.4 \%)$ during that period. Sirolimus was utilized at some point after transplant in 67/278 (24.1\%) patients.

Table 1. Baseline Characteristics of Patients with and without VTE after Lung Transplantation

\begin{tabular}{|c|c|c|}
\hline & VTE $(\mathbf{n}=59)$ & $\begin{array}{c}\text { No VTE } \\
(\mathbf{n = 2 1 9})\end{array}$ \\
\hline \hline Age (years); $\mathrm{p}=0.73$ & $54.4 \pm 12.9$ & $54.9 \pm 8.6$ \\
\hline Type of transplant (single); $=0.84$ & $32(54.2 \%)$ & $122(55.7 \%)$ \\
\hline Pre-transplant diagnoses; $\mathrm{p}=0.28$ & & \\
Emphysema & $24(40.7 \%)$ & $106(48.4 \%)$ \\
Idiopathic pulmonary fibrosis & $22(37.3 \%)$ & $55(25.1 \%)$ \\
Cystic fibrosis & $3(5.1 \%)$ & $16(7.3 \%)$ \\
Sarcoidosis & $1(1.7 \%)$ & $8(3.7 \%)$ \\
Other & $9(15.3 \%)$ & $34(15.6 \%)$ \\
\hline \multicolumn{2}{|c}{}
\end{tabular}

There were 202/278 (72.7\%) patients who were not on chronic anticoagulation, had not received re-transplant or multiple organ transplant, did not have VTE in the first three months after transplant and survived more than three months. Baseline characteristics of patients with and without VTE in this cohort can be seen in Table 2. Sirolimus was utilized in 59/202 (29.2\%) patients. Sirolimus use was more common in patients with VTE $(56.7 \%$ vs. $24.4 \%$; p $<0.001)$.

Table 2. Baseline Characteristics of Lung Transplant Recipients Surviving 90-Days and at-Risk for VTE

\begin{tabular}{|c|c|c|}
\hline & $\begin{array}{c}\text { VTE } \\
(\mathbf{n = 3 0})\end{array}$ & $\begin{array}{c}\text { No VTE } \\
(\mathbf{n = 1 7 2})\end{array}$ \\
\hline \hline Age (years); $\mathrm{p}=0.90$ & $55.1 \pm 9.0$ & $55.3 \pm 8.5$ \\
\hline Type of transplant (single); $\mathrm{p}=0.45$ & $20(33.3 \%)$ & $70(40.7 \%)$ \\
\hline Pre-transplant diagnosis; $\mathrm{p}=0.46$ & & \\
Emphysema & $13(43.3 \%)$ & $100(58.1 \%)$ \\
Idiopathic pulmonary fibrosis & $11(36.7 \%)$ & $43(25.0 \%)$ \\
Cystic fibrosis & $2(6.7 \%)$ & $13(7.6 \%)$ \\
Sarcoidosis & $1(3.3 \%)$ & $5(2.9 \%)$ \\
Other & $3(10.0 \%)$ & $11(6.4 \%)$ \\
\hline Acute rejection score; $\mathrm{p}=0.15$ & $2.53 \pm 3.04$ & $1.69 \pm 2.17$ \\
\hline Acute rejection score per biopsy; & $0.94 \pm 1.01$ & $0.69 \pm 0.85$ \\
$\mathrm{p}=0.22$ & & \\
\hline
\end{tabular}

In further analysis, patients received sirolimus for a significantly lower amount of time compared to other immunosuppression, after the first 3 months were excluded. Total follow-up in patient days was 179335 days on other immunosuppressive medications and 16685 days on sirolimus 


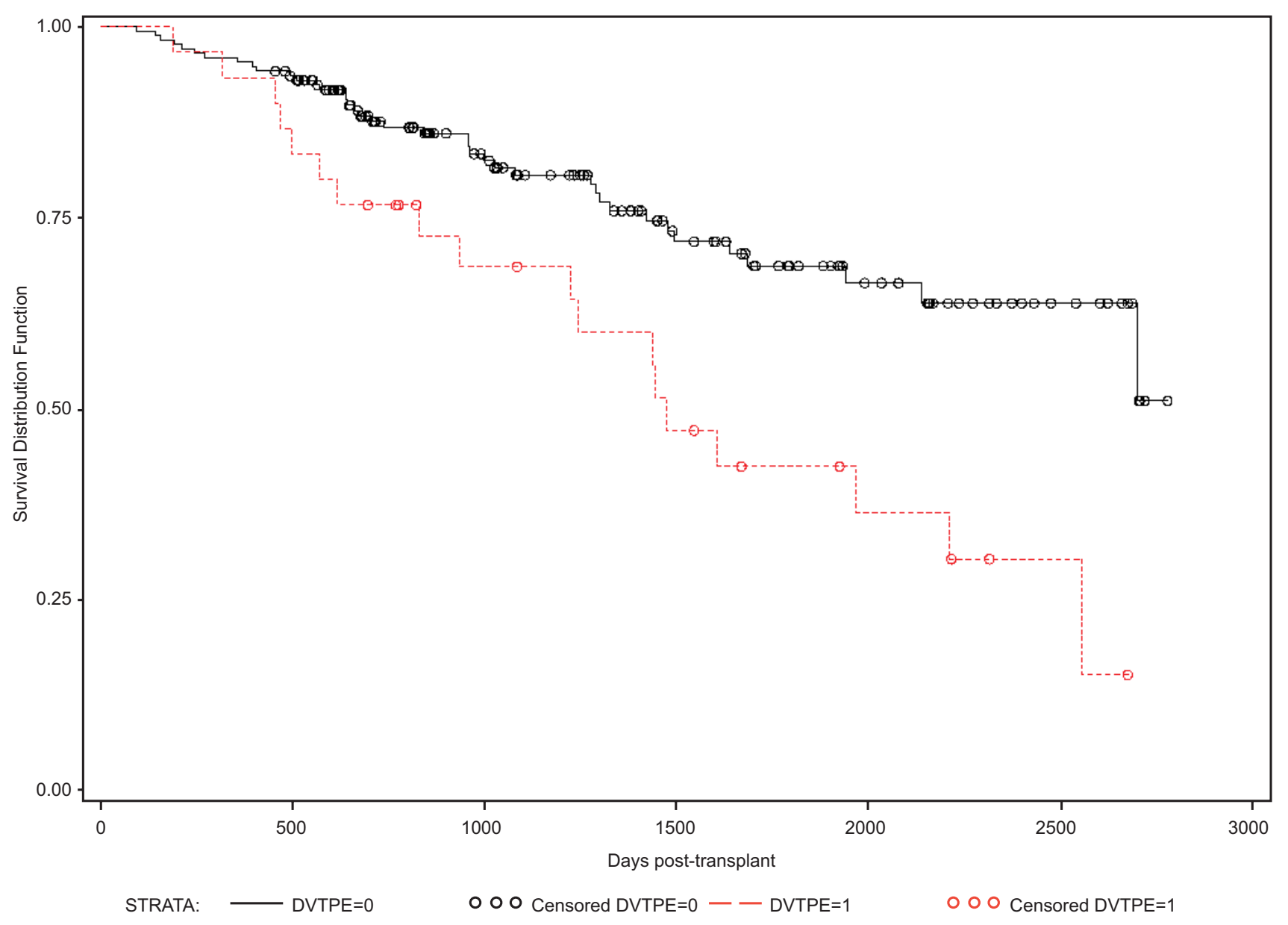

Fig. (1). Survival of patients with and without VTE after lung transplantation excluding the first 90-days post-transplant.

based immunosuppression. The median time on sirolimus was 231 days. When rates of VTE were corrected for time spent on sirolimus or other immunosuppression, rates were significantly higher in patients receiving sirolimus per day spent on the medication: $1.98 \%(1.02 \%$ to $3.40 \%)$ per month of treatment on sirolimus vs. $0.32 \%(0.20 \%$ to $0.48 \%)$ per month on other immunosuppression $(\mathrm{p}<0.001)$. The mean time to VTE on sirolimus was $122 \pm 275$ days.

After multivariable analysis, the major risk factors for development of for VTE, were sirolimus use [odds ratio: $3.79(1.69-8.55)$ ] and increasing ARS [odds ratio: 1.37 (1.08-1.75) for every point increase in ARS].

In the cohort of 3-month survivors, VTE was associated with worse survival as can be seen in Fig. (1) (VTE vs. no VTE: 1-year survival $93.3 \%$ vs. $95.4 \%$; 2-years $76.7 \%$ vs. $87.6 \%$; 3-years $68.6 \%$ vs. $80.6 \%$; 5 -years $42.4 \%$ vs. $68.7 \%$; $\mathrm{p}=0.001$ ). In multivariable analysis development of VTE was the major risk factor for worse survival [hazard ratio: 2.47 (1.41-4.32)] with a diagnosis of idiopathic pulmonary hypertension being a risk factor with borderline significance [hazard ratio: $3.34(0.81-13.8)]$.

\section{DISCUSSION}

VTE is common after lung transplantation. Most of the episodes as expected occur in the early post-transplant period when patients are most likely to be immobile and have recent surgery. However, many episodes occur after the first three months, after the postoperative period. This is similar to prior reported studies [15]. VTE appears to be associated with worse outcomes after lung transplantation. None of the patients in this cohort died as a result of the VTE, however VTE was likely a marker of more severe disease. This was evident when the cohort was restricted to patients that survived at least 3 months after lung transplantation. This is strengthened by the finding that increased ARS was associated with increased risk of VTE.

One of the novel findings in this study was the association of sirolimus with VTE in our patient population. Sirolimus has been used in lung transplant patients since 2001 in select patients. However, early on it was found that sirolimus use in the early post-transplant period was associated with severe complications like anastomotic dehiscence [5]. Its use is therefore rare before the first three months after lung transplantation. Similar complications have been observed in liver transplant recipients (hepatic artery thrombosis), therefore its use in that population is rare [21]. Sirolimus has been used successfully in renal transplant recipients [22]. No excess VTE has been seen in renal transplant recipients, but the trials were not powered to assess that and renal transplant recipients tend to be in lower immunosuppression than lung transplant recipients. In lung transplant recipients, sirolimus is frequently used after 
episodes of acute or chronic rejection, as a rescue therapy. This population tends to be sicker than recipients without acute or chronic rejection. This was evident in our population when acute rejection and sirolimus were both associated with VTE. Some patients received sirolimus as part of an ongoing clinical trial [18] and in this group there were no baseline differences with patients on conventional immunosuppression.

Our study has several limitations. It is a retrospective study, with highly selected patients receiving medications because of multiple reasons that cannot be very easily controlled for. However, the results remained significant after patients that were already on anticoagulation were excluded. VTE was associated with increased risk of poor outcomes after lung transplantation even after controlling for acute rejection and excluding patients that died in the first three months (thus excluding the group with early postoperative complications). Furthermore, it was obvious that even limited exposure to sirolimus led to an increased risk of VTE compared to much longer exposures to other medications. Even though when comparisons with individual patients with VTE off and on sirolimus were not different, this analysis is limited by the rather short time patients remained on sirolimus in general. In addition, our study does not provide a mechanism of how sirolimus might lead to VTE events. It might be a marker of disease rather than the actual culprit for VTE and subsequent poor outcomes. However, the patients had similar baseline characteristics, so there was no obvious upfront selection bias. All these limitations can be overcome by confirming results from randomized controlled clinical trials.

In summary, this study confirms that VTE is common after lung transplantation. A novel finding of our study is that sirolimus appears to be associated with increased risk of VTE events after the early post-transplant period. This risk might be significantly increased compared to patients not on sirolimus. Finally, VTE is associated with worse posttransplant outcomes. The nature of our study makes the conclusions adequate as hypothesis generating, rather than definite. Future prospective studies or increased awareness by other centers will help confirm and further elucidate the mechanism of our findings.

\section{ABBREVIATIONS}

$\begin{aligned} \mathrm{VTE} & =\text { Venous thromboembolism } \\ \mathrm{DVT} & =\text { Deep venous thrombosis } \\ \mathrm{PE} & =\text { Pulmonary embolism } \\ \mathrm{ARS} & =\text { Acute rejection score } \\ \mathrm{BOS} & =\text { Bronchiolitis obliterans syndrome }\end{aligned}$

\section{REFERENCES}

[1] Arcasoy SM, Kotloff RM. Lung Transplantation. N Engl J Med 1999; 340: 1081-91.

[2] Trulock EP, Christie JD, Edwards LB, et al. Registry of the International Society for Heart and Lung Transplantation: twentyfourth official adult lung and heart-lung transplantation report 2007. J Heart Lung Transplant 2007; 26: 782-95.

[3] Mihatsch MJ, Kyo M, Morozumi K, Yamaguchi Y, Nickeleit V, Ryffel B. The side-effects of cyclosporine-A and tacrolimus. Clin Nephrol 1998; 49: 356-63.

[4] Augustine JJ, Bodziak KA, Hricik DE. Use of sirolimus in solid organ transplantation. Drugs 2007; 67: 369-91.

[5] King-Biggs MB, Dunitz JM, Park SJ, Savik SK, Hertz MI. Airway anastomotic dehiscence associated with use of sirolimus immediately after lung transplantation. Transplantation 2003; 75: 1437-43.

[6] Groetzner J, Kur F, Spelsberg F, et al. Airway anastomosis complications in de novo lung transplantation with sirolimus-based immunosuppression. J Heart Lung Transplant 2004; 23: 632-8.

[7] Neff GW, Montalbano M, Tzakis AG. Ten years of sirolimus therapy in orthotopic liver transplant recipients. Transplant Proc 2003; 35: 209S-16S.

[8] Trotter JF. Sirolimus in liver transplantation. Transplant Proc 2003; 35: 193S-200S.

[9] Montalbano M, Neff GW, Yamashiki N, et al. A retrospective review of liver transplant patients treated with sirolimus from a single center: an analysis of sirolimus-related complications. Transplantation 2004; 78: 264-8

[10] Shuchman M. Trading restenosis for thrombosis? New questions about drug-eluting stents. N Engl J Med 2006; 355: 1949-52.

[11] Eisen HJ, Tuzcu EM, Dorent R, et al. Everolimus for the prevention of allograft rejection and vasculopathy in cardiac-transplant recipients. N Engl J Med 2003; 349: 847-58

[12] Langer RM, Kahan BD. Sirolimus does not increase the risk for postoperative thromboembolic events among renal transplant recipients. Transplantation 2003; 76: 318-23.

[13] Kroshus TJ, Kshettry VR, Hertz MI, Bolman RM. Deep venous thrombosis and pulmonary embolism after lung transplantation. J Thorac Cardiovasc Surg 1995; 110: 540-4

[14] Arcasoy SM, Wilt J. Medical complications after lung transplantation. Semin Respir Crit Care Med 2006; 27: 508-520.

[15] Yegen HA, Lederer DJ, Barr RG, et al. Risk factors for venous thromboembolism after lung transplantation. Chest 2007; 132: 547553.

[16] Lingaraju R, Blumenthal NP, Mendez J, et al. Venous Thromboembolic disease after lung transplantation: special focus on sirolimus. Proc Am Thorac Soc 2008; A393 (abstract).

[17] Lingaraju R, Pochettino A, Blumenthal NP, et al. Lung transplant outcomes in white and African american recipients: special focus on acute and chronic rejection. J Heart Lung Transplant 2009; 28 $8-13$.

[18] Bhorade SM, Ahya V, Kotloff RM, et al. Long-term follow-up in the AIRSAC trial, a multicenter randomized clinical trial in lung transplant recipients. J Heart Lung Transplant 2009; 28(Suppl): S154 (abstract).

[19] Sager JS, Kotloff RM, Ahya VN, et al. Association of clinical risk factors with functional status following lung transplantation. Am J Transplant 2006; 6: 2191-2201.

[20] Allison PD. Survival analysis using the SAS system: a practical guide. Cary, NC: SAS Institute, 1995.

[21] Llado L, Fabregat J, Castellote J, et al. Sirolimus-based rescue therapy after rejection in liver transplantation. Clin Transplant 2009; 23: 89-95

[22] Campistol JM, Cockwell P, Diekmann F, et al. Practical recommendations for the early use of m-TOR inhibitors (sirolimus) in renal transplantation. Transplant Int 2009; 22: 681-687. 